\title{
COMPUTING THE BREAKPOINT DISTANCE BETWEEN PARTIALLY ORDERED GENOMES
}

\author{
ZHENG FU AND TAO JIANG \\ Computer Science Department, University of California, Riverside
}

\begin{abstract}
The total order of the genes or markers on a chromosome is crucial for most comparative genomics studies. However, the current gene mapping efforts might only suffice to provide a partial order of the genes on a chromosome. Several different genes or markers might be mapped at the same position due to the low resolution of gene mapping or missing data. Moreover, conflicting datasets might give rise to the ambiguity of gene order. In this paper, we consider the reversal distance and breakpoint distance problems for partially ordered genomes. We first prove that these problems are NP-hard, and then give an efficient heuristic algorithm to compute the breakpoint distance between partially ordered genomes. The algorithm is based on an efficient approximation algorithm for a natural generalization of the wellknown feedback vertex set problem, and has been tested on both simulated and real biological datasets. The experimental results demonstrate that our algorithm is quite effective for estimating the breakpoint distance between partially ordered genomes and for inferring the gene (total) order.
\end{abstract}

\section{Introduction}

The total order of the genes or markers on a chromosome is very important for most comparative genomics studies. The breakpoint distance ${ }^{12,8}$ and reversal distance $e^{11,5}$ are commonly used as the evolutionary distances between genomes, and they work on the premise that the total order of the genes on each chromosome has been identifi ed. However, except for a few model genomes, most genomes have not been completely sequenced yet. For these partially sequenced/assembled genomes, only partial gene maps are available, which might have a low resolution, missing genes/markers, or conflicting ordering information among each other. Combining these partial gene maps together might only suffi ce to provide a partial order of genes and markers. Hence, Zheng, Lenert and Sankoff ${ }^{13,14}$ recently proposed a new general representation of a genome in terms of genes where each chromosome is a directed acyclic graph (DAG) rather than a permutation. Any linearization of the DAGs represents a possible total order of the genome. They generalized the sorting by reversal problem to assess the distance between two partially ordered genomes. The idea is to resolve the partial orders into two total orders (i.e. two linearizations of the DAGs corresponding to the two genomes) with the minimum reversal distance. In the same paper, a depth-fi rst branch-and-bound search algorithm for computing the reversal distance is presented, which runs in exponential time in the worst case.

In this paper, we study effi cient computation of the reversal distance and breakpoint distance problems between two partially ordered genomes. We show that these two problems are NP-hard. We also present an effi cient heuristic algorithm to compute the breakpoint dis- 


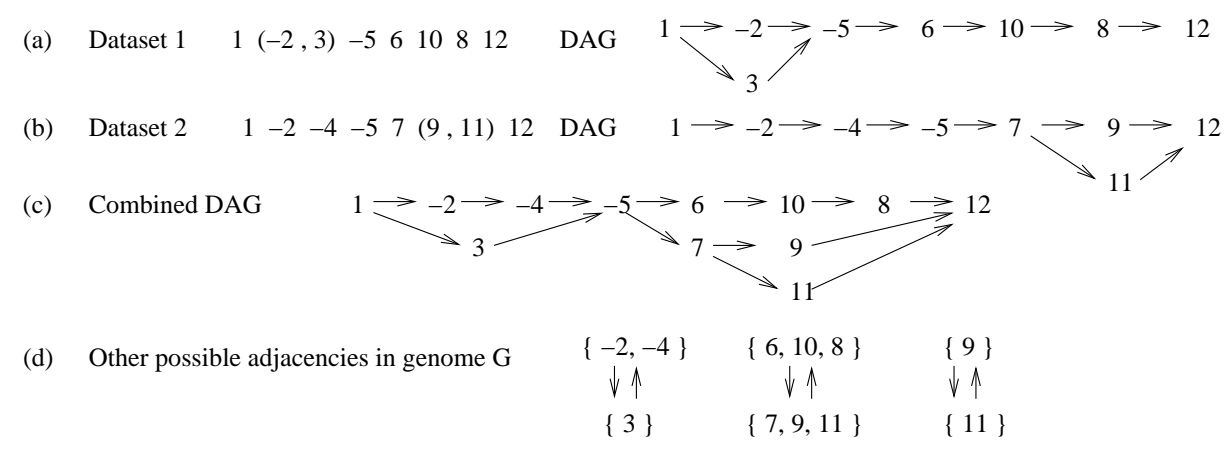

Figure 1. An example of DAG representation for a partially ordered genome.

tance between two partially ordered genomes, called $B D P O G$. The algorithm also reports a pair of total orders for the input genomes realizing the breakpoint distance. It runs in $O\left(n^{3}\right)$ time and uses an effi cient approximation algorithm for a natural generalization of the wellknown feedback vertex set problem as a subroutine. The BDPOG algorithm has been tested on both simulated and real biological datasets. The experimental results demonstrate that it is quite effective for estimating the breakpoint distance between partially ordered genomes and inferring the total gene orders.

The rest of the paper is organized as follows. We fi rst introduce some preliminary facts and defi nitions in Section 2. Section 3 presents the NP-hardness results. Section 4 describes the algorithm BDPOG. Section 5 presents the experimental results on both simulated and real genome datasets. Finally, some concluding remarks are given in Section 6.

\section{Preliminaries}

Genes or markers are usually represented by signed (+ or -) symbols from a alphabet $A$, where the signs represent the strand of the genes. A totally ordered genome could be modeled as an ordered string of genes. However, the existing gene mapping efforts might only suffi ce to partially order the set of genes on a chromosome. If the order of some genes (e.g. $\left.a_{1}, a_{2}, \cdots, a_{n}\right)$ cannot be decided in a gene map, we will use $\left(a_{1}, a_{2}, \cdots, a_{n}\right)$ to represent the uncertainty of the ordering among them. For example, in the gene map $1(-2,3)-5610812$, the ordering of all the genes has been decided except between genes 2 and 3 .

Two or more gene maps constructed from different kinds of data or using different methodologies can be combined to form a more complicated partial order. As Zheng, Lenert, and Sankoff proposed in recent studies ${ }^{13,14}$, directed acyclic graphs (DAGs) rather than linear permutations could be used to represent partially ordered genomes. In each DAG, all genes are represented by vertices, while the ordering relation between the genes is represented by arcs (see Figure 1).

Let $\Pi$ and $\Gamma$ be partially ordered genomes of size $n$, and the DAG representations for $\Pi$ and $\Gamma$ denoted as $\mathrm{DAG}(\Pi)$ and $\mathrm{DAG}(\Gamma)$. A linearization of $\mathrm{DAG}(\Pi)$ represents a possible ordering of genome $\Pi$. Let $L(\Pi)$ be the set of all possible linearizations of the $\mathrm{DAG}(\Pi)$. 
Then we defi ne the reversal distance between $\Pi$ and $\Gamma$ as

$$
d_{r}(\Pi, \Gamma)=\min _{\pi \in L(\Pi), \gamma \in L(\Gamma)} d_{r}(\pi, \gamma)
$$

Similarly, the breakpoint distance is

$$
d_{b}(\Pi, \Gamma)=\min _{\pi \in L(\Pi), \gamma \in L(\Gamma)} d_{b}(\pi, \gamma)
$$

We defi ne the problem of computing $d_{r}(\Pi, \Gamma)$ as the partial-order reversal distance $(P R D)$ problem and the problem of computing $d_{b}(\Pi, \Gamma)$ as the partial-order breakpoint distance $(P B D)$ problem.

Clearly, all possible pairwise adjacency relationships in all possible linearizations of a DAG can be represented by the arcs of a DAG plus two arcs of opposite directions between all pairs of vertices which are not ordered by the DAG (see Figure 1d). We say that a pair of genes forms a possible adjacency in genome $\Pi$ if they are possibly adjacent in any linearization of $\mathrm{DAG}(\Pi)$. We say that a pair of genes $a$ and $b$ is a possible common adjacency and write $a \cdot b$ if they are a possible adjacency in both genomes $\Pi$ and $\Gamma$. And $a$ is the left end of $a \cdot b$ and $b$ is the right end of $a \cdot b$. Let $\mathcal{S}$ be the set of all possible common adjacencies. Defi ne an order relation " $\leadsto$ " between a pair of possible common adjacencies $a \cdot b$ and $c \cdot d$ in $\mathcal{S}$. We write $a \cdot b \leadsto \Pi c \cdot d$ if one of the following four conditions is satisfi ed: (i) $c$ or $d$ is reachable from $a$ or $b$ in $\operatorname{DAG}(\Pi)$ (i.e. at least one of the genes in the second possible common adjacency is reachable from at least one of the genes in the first possible common adjacency in DAG(II)); (ii) $a=c$ and $b \neq d$, or $b=d$ and $a \neq c$ (i.e. two different possible common adjacencies share the same left end or the same right end); (iii) $b=c$ (i.e. the right end of the fi rst possible common adjacency is the same as the left end of the second possible common adjacency); (iv) let $a=u_{m-1}, b=u_{m}, c=u_{1}$ and $d=u_{2}$, and there exist possible common adjacencies $u_{1} \cdot u_{2}, u_{2} \cdot u_{3}, \cdots, u_{m-1} \cdot u_{m}, 3 \leq m$ and another path in DAG(П) from $u_{1}$ to $u_{m}$ other than $u_{1} \rightarrow u_{2} \rightarrow \cdots \rightarrow u_{m-1} \rightarrow u_{m}$.

Based on the order relation " $\sim \Pi$ ", we defi ne a directed graph $\mathcal{G}_{\Pi}$, called the adjacencyorder graph. The construction of $\mathcal{G}_{\Pi}$ is described as follows (see fi gure 2):

- Every possible adjacency in $\mathcal{S}$ is represented by a vertex.

- For every two possible common adjacencies, if $a \cdot b \sim_{\Pi} c \cdot d$, add an arc from the vertex $a \cdot b$ to the vertex $c \cdot d$.

A directed cycle in an adjacency-order graph usually represents a conflict among the possible common adjacencies in this cycle. And based on the construction of the adjacencyorder graph, we have the following theorem.

Theorem 2.1. All the possible common adjacencies in an acyclic adjacency-order graph $\mathcal{G}_{\Pi}$ could always co-exist in some linearization of $D A G(\Pi)$.

Proof. Omitted due to page limit. Please see the full version.

The graph $\mathcal{G}_{\Gamma}$ can be constructed in the same way except that the arcs represent the relation $\leadsto \Gamma$ instead of $\leadsto \Pi$. Note that since the adjacency-order graphs $\mathcal{G}_{\Pi}$ and $\mathcal{G}_{\Gamma}$ are 
(a)

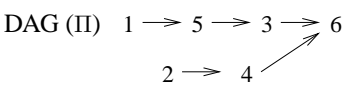

(b) Possible common adjacency set $S$ :

(c)

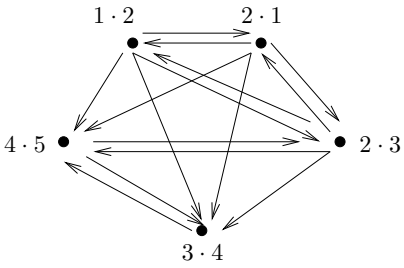

Adjacency-order graph $\mathcal{G}_{\Pi}$

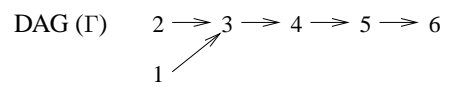

$\{1 \cdot 2,2 \cdot 1,2 \cdot 3,3 \cdot 4,4 \cdot 5\}$

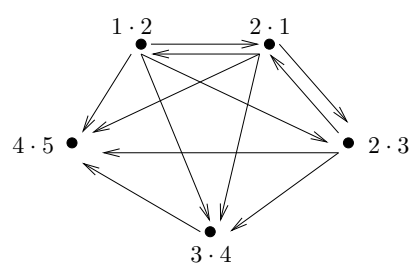

Adjacency-order graph $\mathcal{G}_{\Gamma}$

Figure 2. An example of the construction of adjacency-order graphs. In $\mathcal{G}_{\Pi}$, the arcs inserted by condition (i): $(1 \cdot 2,2 \cdot 3),(1 \cdot 2,3 \cdot 4),(1 \cdot 2,4 \cdot 5),(2 \cdot 1,2 \cdot 3),(2 \cdot 1,3 \cdot 4),(2 \cdot 1,4 \cdot 5),(2 \cdot 3,3 \cdot 4),(2 \cdot 3,4 \cdot 5)$, $(4 \cdot 5,2 \cdot 3),(4 \cdot 5,3 \cdot 4)$; the arcs inserted by condition (ii): $(2 \cdot 1,2 \cdot 3),(2 \cdot 3,2 \cdot 1)$; the arcs inserted by condition (iii): $(1 \cdot 2,2 \cdot 1),(1 \cdot 2,2 \cdot 3),(2 \cdot 1,1 \cdot 2),(2 \cdot 3,3 \cdot 4),(3 \cdot 4,4 \cdot 5)$; and the arc inserted by condition (iv): $(2 \cdot 3,1 \cdot 2)$. Note that some arcs might satisfy several different conditions.

constructed by possible common adjacencies, they should share a same vertex set but may have different arc sets.

\section{Computational Complexity of the PRD and PBD Problems}

In this section, we show that both PRD and PBD problems are NP-hard, using different reductions.

\section{Theorem 3.1. The PRD problem is NP-hard.}

Proof. The proof is based on a careful analysis of the structure of the breakpoint graph for two partially ordered genomes ${ }^{13,14}$, the Hannenhalli-Pevzner formula ${ }^{5}$ for the reversal distance between two totally ordered genomes, and a reduction from the NP-hard problem MAX-ACD ${ }^{2}$. The details are omitted due to page limit. Please see the full version.

By using a different reduction, we can prove the NP-hardness of the the breakpoint distance between two partially ordered genomes.

Theorem 3.2. The PBD problem is NP-hard.

Proof. We prove that the decision version of the PBD problem is NP-hard by a reduction from the decision version of minimum feedback vertex set problem.

Minimum Feedback Vertex Set Problem (MFVS)

INSTANCE: A directed graph $G(V, A)$ and a positive integer $k$.

QUESTION: Is there a subset $X \subseteq V$ with $|X| \leq k$ such that deleting all the vertices from $X$ and their incident arcs will leave $G$ acyclic?

Let directed graph $G(V, A)$ and positive integer $k$ make up an arbitrary instance of the MFVS problem. The reduction to the breakpoint distance problem between partial ordered permutations ( $\Pi$ and $\Gamma$ ) works as follows: (a) For every vertex $v_{i}$ in $G$, make two genes $v_{i}^{1}$ 
and $v_{i}^{2}$. (b) Add another $n+1$ genes $\left\{x_{1}, x_{2} \cdots, x_{n+1}\right\}$, where $n=|V|$. (c) Construct a totally ordered genome $\Gamma=x_{1} v_{1}^{1} v_{1}^{2} x_{2} v_{2}^{1} v_{2}^{2} \cdots x_{n} v_{n}^{1} v_{n}^{2} x_{n+1}$. (d) Construct a partially ordered genome $\Pi=x_{n+1}\left(p_{1}, p_{2}, \cdots, p_{m}\right) x_{1} \cdots x_{n}$, where $m=|E|$ and each $p_{i}, i \in[1, m]$, represents an ordered pair of vertices. If there is an arc directed from vertex $v_{u}$ to vertex $v_{w}$ in $G$, we will have a pair $p_{i}=v_{u}^{1} v_{w}^{2}$, which means that in the genome $\Pi$ gene $v_{u}^{1}$ is ordered before gene $v_{w}^{2}$. Finally, the order between $p_{i}$ and $p_{j}, i \neq j$, is unknown. Figure 3 gives a simple example for this reduction.

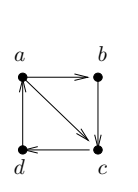

(a)

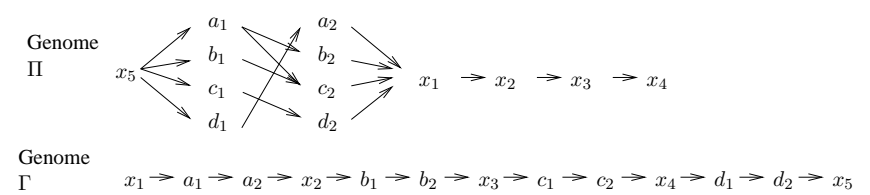

(b)

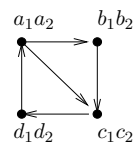

(c)

Figure 3. An example of the reduction from the minimum feedback vertex set problem to the breakpoint distance problem. (a) Directed graph $G(V, A)$. (b) Genome $\Pi$ and $\Gamma$, where $\Gamma$ is a totally ordered genome. (c) Adjacencyorder graph of $\Pi, \mathcal{G}_{\Pi}$, which is isomorphic to $G(V, A)$.

This reduction guarantees that for $\Pi$ and $\Gamma$, the set of all possible common adjacencies $\mathcal{S}=\left\{v_{1}^{1} \cdot v_{1}^{2}, v_{2}^{1} \cdot v_{2}^{2}, \cdots, v_{n}^{1} \cdot v_{n}^{2}\right\}$. Adjacency-order graph $\mathcal{G}_{\Pi}$ is isomorphic to $G(V, A)$, while adjacency-order graph $\mathcal{G}_{\Gamma}$ is acyclic since $\Gamma$ is totally ordered. Based on the special construction of $\Pi$ and $\Gamma$, the cardinality of the minimum feedback vertex set of $\mathcal{G}_{\Pi}$, or graph $G(V, A)$, is exactly $d_{b}(\Pi, \Gamma)-2 n-2$. Therefore, the feedback vertex set problem of $G(V, A)$ and $k$ could be resolved by computing the $d_{b}(\Pi, \Gamma)$. The result of Theorem 3.2 hence follows.

\section{An Efficient Heuristic Algorithm for Computing the Breakpoint Distance}

Let $\Pi$ and $\Gamma$ be two partially ordered genomes with possible common adjacency set $\mathcal{S}$. Computing the breakpoint distance $d_{b}(\Pi, \Gamma)$ is actually the problem of finding two linearizations of $\Pi$ and $\Gamma$ containing the maximum number of possible common adjacencies. In other words, we want to delete the smallest number of possible common adjacencies from $\mathcal{S}$ while leaving the rest of possible common adjacencies conflict free (i.e. they could co-exist in some linearizations). One way to delete order conflicts among possible common adjacencies is using the adjacency-order graph.

By Theorem 2.1, if the adjacency-order graph is acyclic, all the possible common adjacency vertices could be linearized by topological sort and partially ordered genomes could be totally ordered based on such a topological sort. Hence, deleting the smallest number of vertices to make both adjacency-order graphs (i.e. $\mathcal{G}_{\Pi}$ and $\mathcal{G}_{\Gamma}$ ) acyclic simultaneously could approximate the $d_{b}(\Pi, \Gamma)$. Formally,

Definition 4.1. Minimum Double Feedback Vertex Set (MDFVS) problem

Given two directed graphs with the same vertex set and different arc sets, fi nd the minimumcardinality subset of the vertices whose deletion leaves both graphs acyclic simultaneously. 
The output vertex set is called a minimum double feedback vertex set.

\subsection{An Efficient Approximation Algorithm for the Minimum Double Feedback Vertex Set Problem}

Recall that the minimum feedback vertex set (MFVS) problem deals with a single graph, i.e., the goal is to find the subset of vertices with the minimum cardinality whose deletion will leave the (single) input graph acyclic. We know that for the minimum feedback vertex set problem, the best-known approximation algorithm ${ }^{4,10}$ in directed graphs achieves a performance ratio of $O(\log n \log \log n)$, where $n$ is the number of vertices of the digraph, although the algorithm requires to the solution of a linear program. Another useful approximation algorithm ${ }^{3}$ (denoted APPROX-MFVS) achieves a performance ratio bounded by the length, in terms of the number of vertices, of a longest simple cycle in the input digraph. Based on the strong relationship between the MFVS problem and the MDFVS problem, we could prove the following theorem.

Theorem 4.1. There exists a polynomial $2 \lambda$-approximation algorithm for the MDFVS problem, where $\lambda$ is the maximum length, in terms of the number of vertices, of a longest simple cycle in any of the two input graphs.

Proof. In the MDFVS problem, we are given two directed graphs, say $G_{1}$ and $G_{2}$, which have the same vertex set and different arc sets. Utilizing the approximation algorithm APPROX-MFVS for the MFVS problem as a subroutine, we can easily design an approximation algorithm, denoted APPROX-MDFVS (see Figure 4), for the MDFVS problem as follows. Run APPROX-MFVS on $G_{1}$ and $G_{2}$ separately to get the feedback vertex sets $\operatorname{FVS}\left(G_{1}\right)$ and $\operatorname{FVS}\left(G_{2}\right)$, respectively. Denote the union of $\operatorname{FVS}\left(G_{1}\right)$ and $\operatorname{FVS}\left(G_{2}\right)$ as $\operatorname{DFVS}\left(G_{1}, G_{2}\right)$. DFVS $\left(G_{1}, G_{2}\right)$ is certainly a double feedback vertex set, although not necessarily minimal. In fact, it might contain some vertices whose deletion will not affect the property of DFVS. Hence, the algorithm in its last step greedily removes vertices from $\operatorname{DFVS}\left(G_{1}, G_{2}\right)$ as much as possible, as long as the remaining vertices still form a DFVS. Let $\mathrm{OPT}_{1}$ and $\mathrm{OPT}_{2}$ be the optimal values of MFVS on $G_{1}$ and $G_{2}$ respectively. Let OPT be the optimal value of MDFVS on $G_{1}$ and $G_{2}$. It is obvious that $\mathrm{OPT}_{1}, \mathrm{OPT}_{2} \leq$ OPT. Since $\left|\operatorname{FVS}\left(G_{1}\right)\right| \leq \lambda_{1} \mathrm{OPT}_{1}$, where $\lambda_{1}$ is the length of a longest simple cycle in $G_{1}$, and $\left|\operatorname{FVS}\left(G_{2}\right)\right| \leq \lambda_{2} \mathrm{OPT}_{2}$, where $\lambda_{2}$ is the length of a longest simple cycle in $G_{2}$, we get $\operatorname{DFVS}\left(G_{1}, G_{2}\right) \leq 2 \lambda$ OPT, where $\lambda=\max \left\{\lambda_{1}, \lambda_{2}\right\}$. Since the algorithm APPROXMFVS can be implemented in $O\left(n^{3}\right)$ worst-case running time, the algorithm APPROXMDFVS also runs in $O\left(n^{3}\right)$ time.

\subsection{The Final Heuristic Algorithm for Breakpoint Distance}

Following the above discussion, we present an effi cient heuristic algorithm, denoted as BDPOG, to calculate $d_{b}(\Pi, \Gamma)$ in four steps, given DAG $(\Pi)$ and $\mathrm{DAG}(\Gamma)$ :

(1) Add two vertices (e.g. $v_{0}$ and $v_{n+1}$ ) to the two input DAGs. In each DAG, add arcs from $v_{0}$ to all the vertices with in-degree 0 , and add arcs from all the vertices with 


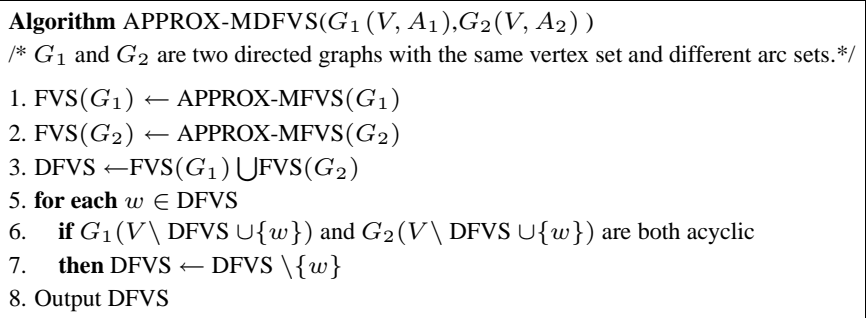

Figure 4. The approximation algorithm for MDFVS.

out-degree 0 to $v_{n+1}$.

(2) Derive the possible common adjacency set $\mathcal{S}$ from the DAGs and construct the adjacency-order graphs $\mathcal{G}_{\Pi}$ and $\mathcal{G}_{\Gamma}$.

(3) Find a double feedback vertex set for $\mathcal{G}_{\Pi}$ and $\mathcal{G}_{\Gamma}$, denoted as $\operatorname{DFVS}\left(\mathcal{G}_{\Pi}, \mathcal{G}_{\Gamma}\right)$, by applying the APPROX-MDFVS algorithm.

(4) Output $n+1-|\mathcal{S}|+\left|\operatorname{DFVS}\left(\mathcal{G}_{\Pi}, \mathcal{G}_{\Gamma}\right)\right|$ as $d_{b}(\Pi, \Gamma)$ and the corresponding total orders of $\Pi$ and $\Gamma$.

It is obvious that the performance of the BDPOG algorithm directly depends on the performance of the APPROX-MDFVS algorithm. The construction of the adjacency-order graphs in step 2 takes $O\left(n^{3}\right)$ time, where $n$ is the total number of genes, since it involves a transitive closure construction. Since the APPROX-MDFVS algorithm runs in $O\left(n^{3}\right)$ time, the overall running time of the BDPOG algorithm is $O\left(n^{3}\right)$.

\section{Experimental Results}

In order to test the performance of the BDPOG algorithm, we have applied it to both simulated data and real biological data. We will also use an example from the Comparative Grass Genomics database (http://www.gramene.org) to illustrate the application of our method on real data.

\subsection{Simulated Data}

We use simulated data to assess the performance of our algorithm on computing the breakpoint distance between two partially ordered genomes. The simulated data is generated as follows. Start from a genome $G$ with $n$ distinct symbols whose signs are generated randomly. Perform $r$ reversals on the genome $G$ to obtain another genome $H$. The boundaries of these reversals are uniformly distributed within the range of the genome. The maps of these two simulated genomes are generated according to two parameters: the group rate $p$ corresponding to the probability of a gene being placed at the same position as the next gene, and the missing rate $q$ that determines how many genes are missing from the map. Each gene is subjected independently to these two events. Note that every gene has to exist in at least one map of each genome. Then we combine all the map datasets for each genome into a DAG. Clearly, these two DAGs represents two partially ordered genomes $g$ 
and $h$ generated from genomes $G$ and $H$. The quadruple $(n, r, p, q)$ specifi es the parameters for generating two partially ordered genomes as test data.

We run BDPOG on 20 random instances for each combination of parameters. The average breakpoint distance between partially ordered genomes $g$ and $h$, computed by BDPOG, is compared with the average breakpoint distance between totally ordered genomes $G$ and $H$. The results are shown in Figure 5. As we can see from the fi gure, our heuristic algorithm is quite reliable in computing the breakpoint distance between two partially ordered genomes. On average, the distance computed by BDPOG algorithm is very close to the real breakpoint distance between the totally ordered genomes. The difference between two breakpoint distances generally increases as two genomes become more related, or the uncertainty of gene orders increases, e.g., increasing $(p, q)$ from $(0.2,0.1)$ to $(0.4,0.2)$.
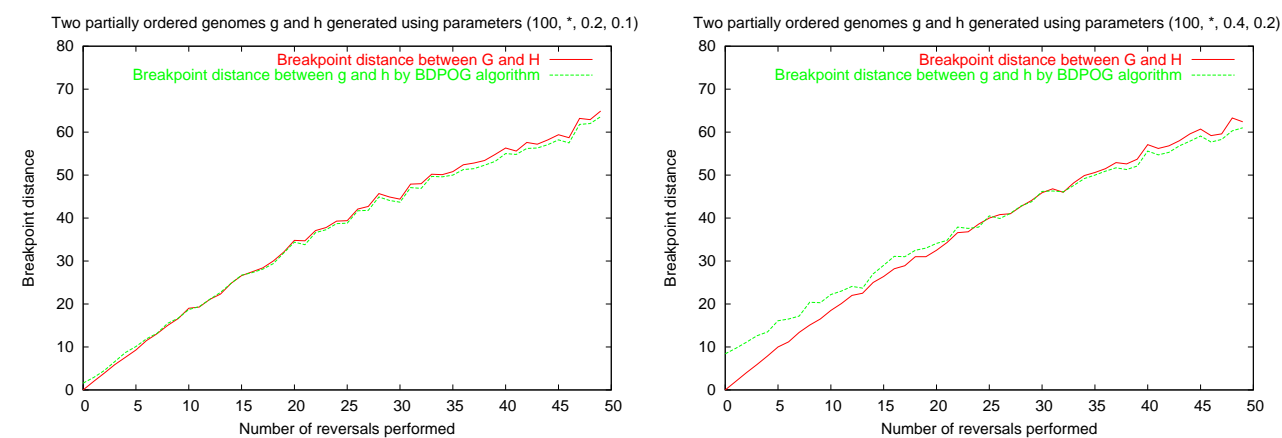

Figure 5. Performance of our heuristic algorithm BDPOG on simulated data.

\subsection{Real Data}

We use the X chromosomes of human (Homo sapiens, UCSC hg18, March 2006), mouse (Mus musculus, UCSC mm8, March 2006), and rat (Rattus norvegicus, UCSC rn4, November 2004) genomes in our real data test. In these three datasets downloaded from the UCSC Genome Browser ${ }^{6}$ website (http://genome.ucsc.edu), all the genes are totally ordered. We perform the test between each pair of genomes, where we extract all the gene orthologs between the two compared genomes. Then we generate two partially ordered genomes for the compared genomes using the method described in the previous section, although we need to specify the group rate $p$ and missing rate $q$ here. By using our heuristic algorithm, the breakpoint distance between the simulated partially ordered genomes and the possible total order for each genome can be determined. We run our heuristic algorithm BDPOG on ten random instances, and compare the average estimated breakpoint distance and the gene orders with the real ones. The results are shown in Table 1. For example, if we generate the partially ordered chromosomes for the $\mathrm{X}$ chromosomes of human and mouse by using parameters $p=0.2$ and $q=0.1$, we get 44.15 as the average estimated breakpoint distance. In the total gene orders output by our algorithm, an average of 384.05 gene adjacencies 
among 388 are kept for the human $\mathrm{X}$ chromosome and an average of 382.9 gene adjacencies among 388 are kept for the mouse $\mathrm{X}$ chromosome. Note that, the average estimated breakpoint distance 44.15 is smaller than the real breakpoint distance between human and mouse, i.e., 45. A possible reason is that a small amount of uncertainties in gene order might actually decrease the number of reversals between two genomes. Overall, the results demonstrate that our algorithm performs very well on estimating breakpoint distance and recovering the gene orders for partially ordered genomes.

Table 1. Comparison of the estimated breakpoint distances and the gene orders with the real ones. $\zeta$ The number of the common gene adjacencies exist in both the real genome $G$ and the total order of the partially ordered genome $g$ obtained by BDPOG.

\begin{tabular}{|c|c|c|c|c|c|c|c|c|}
\hline \multirow{2}{*}{$G / H$} & \multirow{3}{*}{} & \multirow{3}{*}{ \#orthologs } & \multirow{2}{*}{$d_{b}(G, H)$} & \multicolumn{3}{|c|}{$g$ and $h(p=0.2, q=0.1)$} & \multicolumn{3}{|c|}{$g$ and $h(p=0.4, q=0.2)$} \\
\cline { 4 - 9 } & & & $\begin{array}{c}\text { estimated } \\
d_{b}(g, h)\end{array}$ & $\begin{array}{c}\text { common adjs } \\
\text { in } g \text { and } G\end{array}$ & $\begin{array}{c}\text { common adjs } \\
\text { in } h \text { and } H\end{array}$ & $\begin{array}{c}\text { estimated } \\
d_{b}(g, h)\end{array}$ & $\begin{array}{c}\text { common adjs } \\
\text { in } g \text { and } G\end{array}$ & $\begin{array}{c}\text { common adjs } \\
\text { in } h \text { and } H\end{array}$ \\
\hline human/mouse & 389 & 45 & 44.15 & 384.05 & 382.9 & 63.75 & 380.3 & 379.4 \\
human/rat & 132 & 22 & 21.3 & 129.9 & 131 & 27.2 & 128.5 & 126.4 \\
mouse/rat & 126 & 17 & 15.65 & 124.3 & 124.05 & 20 & 123.45 & 121.95 \\
\hline
\end{tabular}

To further illustrate the application of our method on real data, we use an example from the Comparative Grass Genomics database (http://www.gramene.org). We examine two closely related genomes, maize and sorghum. We used the "IBM2 neighbors 2004" and the "IBM neighbors maps" 9 for chromosome 1 of maize, and compared it with the "Paterson 2003" ${ }^{1}$ and the "Klein 2004" 7 maps for the chromosome labeled $C$ and $L G-01$, respectively, of sorghum. All markers of maize indicated as having a homolog in one of the datasets of sorghum are extracted, and vice versa. We extracted 21 markers in total. The two DAGs constructed from the maize datasets and sorghum datasets and the total order of the DAGs output by our algorithm are shown in Figure 6.

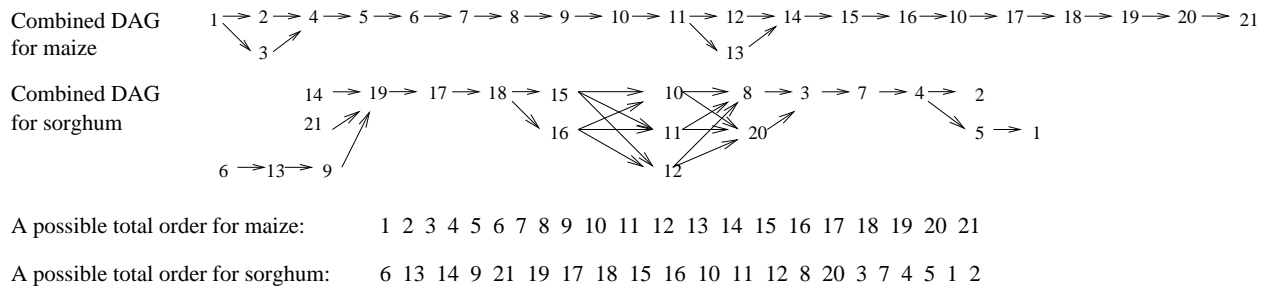

Figure 6. A comparison of maize and sorghum chromosomes using partially ordered data from the Gramene database.

\section{Conclusion}

In this paper, we have presented some complexity and algorithmic results for the problem of comparing two partially ordered genomes. In particular, we proposed an effi cient heuris- 
tic algorithm to estimate the breakpoint distance between two partially ordered genomes and infer the corresponding linearizations achieving the distance. In our construction, we defi ned a useful tool, called the adjacency-order graph, and introduced a new optimization problem (MDFVS), for which we designed an effi cient approximation algorithm. Our preliminary experiments on simulated and real data have demonstrated that our algorithm performs very well on estimating breakpoint distance and recovering the gene orders for partially ordered genomes. Considering the breakpoint distance is just the fi rst step. In the future, we plan to look into other distances between partially ordered genomes, e.g., the reversal distance, and try to design more effi cient algorithms.

\section{Acknowledgement}

This project is supported in part by NSF grant CCR-0309902, National Key Project for Basic Research (973) grant 2002CB512801, NSFC grant 60528001, and a Changjiang Visiting Professorship at Tsinghua University.

\section{References}

1. J.E. Bowers et al., "A high-density genetic recombination map of sequence-tagged sites for sorghum, as a framework for comparative structural and evolutionary genomics of tropical grains and grasses," Genetics, 165:367-386, 2003.

2. A. Caprara, "Sorting by reversal is diffi cult," Proc. 1St RECOMB, pp.75-83, 1997.

3. C. Demetrescu and I. Finocchi, "Combinatorial Algorithms for Feedback Problems," Information Processing Letters, 86(3):129-136, 2003.

4. G. Even et al., "Approximating minimum feedback sets and multi-cuts in directed graphs," Proc. 4th Int. Conf. on Integer Prog. and Combinatorial Optimization, Lecture Notes in Comput. Sci., 920, Springer-Verlag, 14-28, 1995.

5. S. Hannenhalli and P. Pevzner, "Transforming cabbage into turnip (Polynomial algorithm for sorting signed permutations by reversals)," Proc. of 27th Annual ACM Symposium on the Theory of Computing, pp.178-187, 1995.

6. D. Karolchik et al., "The UCSC Genome Browser Database," Nucleic Acids Res., 31(1): 51-54, 2003.

7. M.A. Menz et al., "A high-density genetic map of Sorghum bicolor (L.) Moench based on 2926 AFLP, RFLP and SSR markers," Plant molecular biology 48:483-499, 2002

8. J. Nadeau and B. Taylor, 'Lengths of chromosomal segments conserved since divergence of man and mouse," Proc. Natl. Acad. Sci., 81:814-818, 1984.

9. M.L. Polacco and Jr Coe E., 'IBM neighbors: a consensus GeneticMap," 2002

10. P.D. Seymour, 'Packing directed circuits fractionally," Combinatorica, 15:281-288, 1995.

11. D. Sankoff, 'Mechanisms of genome evolution: models and inference,' Bull. Int. Stat. Institut., 47:461-475, 1989.

12. G. Watterson et al., "The chromosome inversion problem," J. Theor. Biol., 99:1-7, 1982.

13. C. Zheng and D. Sankoff, "Genome rearrangements with partially ordered chromosomes," $\mathrm{CO}$ COON, 2005.

14. C. Zheng, A. Lenert, and D. Sankoff, 'Reversal distance for partially ordered genomes," Bioinformatics, 21(Suppl.1):i502-i508, 2005. 